\title{
Histología y morfometría de piel del pez Eremophilus mutisii (Trychomecteridae, Siluriformes)
}

\author{
Rocío Johanna Bonilla Lizarazo, Marllury Quintero Virguez, Edwin Gómez Ramírez, Daniel \\ Rodríguez Caicedo \& Hernán Hurtado Giraldo \\ Laboratorio de Histoembriología, Grupo de Ictiología, Programa de Biología Aplicada, Facultad de Ciencias, \\ Universidad Militar Nueva Granada. Cra 11 101-80 Bogotá, Colombia; hhurtado@umng.edu.co
}

\author{
Recibido 22-XII-2006. Corregido 30-IX-2007. Aceptado 28-IV-2008.
}

\begin{abstract}
Skin histology and morphometry of the fish Eremophilus mutisii (Trychomecteridae, Siluriformes). The tropical freshwater fish Eremophylus mutisii is endemic to the Cundinamarca highland in Colombia. Skin samples $\left(0.5 \times 0.5 \mathrm{~cm}^{2}\right)$ were taken from 11 specimens at six body parts (mandible, dorsal head, dorsal trunk, caudal trunk, medial trunk and abdominal area), fixed in $4 \%$ formaldehyde, dehydrated in $95 \%$ ethanol and $99 \%$ isopropanol, embedded in paraffin and sectioned at $5 \mu \mathrm{m}$. The skin is made of two mayor cutaneous layers (epidermis and dermis) and a subcutaneous layer (hypodermis). The epidermis presents three layers with secretory cells, epithelial cells and a few taste buds; the dermis is separated from the epidermis by a basal membrane. We observed fibroblasts, two layers of melanophors and some blood vessels; the hypodermis has vascularized adipose tissue. Skin thickness changes with body area; the dermis is thicker than the epidermis; skin has more club cells than mucous cells. The medial trunk area has the largest number of club and mucous cells. The skin of E. mutissi seems to mainly have a protective function. Rev. Biol. Trop. 56 (2): 885-893. Epub 2008 June 30.
\end{abstract}

Key words: Eremophylus mutissi, skin, histology, morphometry.

El pez llamado en Colombia Capitán de la Sabana, Eremophilus mutisii, es endémico de las aguas naturales fluviales y lacustres del altiplano Cundiboyacense (Borissow y Canosa 2000, Rodríguez 2000). Aunque ya se conocen algunos trabajos sobre aspectos ecológicos y reproductivos de esta especie (e.g Cala et al. 1990, Rodríguez 1991, Mayorga 1992, Jiménez y Pinto 2005), la información sobre la estructura de la piel de E. mutisii a nivel histológico, es muy escasa (Bonilla et al. 2005). Por la función que se atribuye a la piel como barrera entre el pez, al medio en que habita (fangoso y turbio), y al reducido tamaño de sus ojos, es probable que gran parte de la interacción de E. mutisii con el ambiente se haga a través de la piel. Por esta razón se planteó este estudio con el ánimo de aportar información a la biología básica de este pez, caracterizando histológicamente y morfométricamente la estructura de la piel, para reconocer los mecanismos de protección y recepción sensorial para mantenerse en este tipo de hábitat.

La piel es el límite entre el cuerpo y el ambiente. Según Tyagi y Shukla (2002) una de las principales funciones de la piel es de protección, ya que el mucus secretado por las glándulas mucosas cubre el cuerpo del pez, protegiéndolo contra bacterias, hongos y otros microorganismos. Adicionalmente, lo lubrica reduciendo la fricción corporal en el agua mientras nada, esto le permite alcanzar grandes velocidades. La piel cumple una función importante en la reparación de heridas superficiales, inmediatamente después de una lesión la herida es cerrada por el mucus el cual 
contiene un gran número de linfocitos. Las células marginales de la herida pueden multiplicarse rápidamente para formar una delgada capa protectora y progresivamente se completa la curación de la herida.

La piel es delgada (Tyagi y Shukla 2002). Consta de dos capas (epidermis y dermis), además de una capa hipodérmica subcutánea. Según Elliott (2000) la epidermis de los peces consta de un epitelio plano estratificado no queratinizado. El número de capas celulares puede variar de dos en las larvas hasta diez o más en los adultos. En las especies pelágicas, la epidermis es frecuentemente más gruesa en las áreas dorsales del cuerpo, mientras que las especies bentónicas la epidermis presenta mayor grosor en las superficies que cubren la parte ventral. En general la epidermis de los peces presenta diferentes tipos de células como células epiteliales entre las cuales se encuentran regularmente células mucosas, células clava de gran tamaño, células sensoriales (botones gustativos, neuromastos), fibras nerviosas (Grazzle 1976, Ottensen y Olafsen 1997, Elliott 2000, Webb 2000, Stone et al. 1995). La dermis está compuesta por tejido conjuntivo denso o fibroso; posee algunas capas de células de pigmento en la parte marginal entre la epidermis y la capa subcutánea (hipodermis), las cuales dan el color a los peces, macrófagos y mastocitos (Elliott 2000, Souza et al. 2003, Le Guellec 2004). La hipodermis separa la parte interna de la dermis del músculo subyacente (Le Guellec 2004), es considerada como una variante del tejido conjuntivo, especializado en el almacenamiento de lípidos, cuyas células principales son los adipocitos, que se encuentran en una malla de tejido conjuntivo reticular, en el cual es posible observar capilares sanguíneos.

\section{MATERIALES Y MÉTODOS}

La captura de los ejemplares, se llevó a cabo en el periodo comprendido durante octubre de 2002 - mayo de 2004, en un tramo del Río Bogotá (aproximadamente $150 \mathrm{~m}$ ) en la vereda Cacicazgo aledaña al municipio de
Suesca, Cundinamarca, Colombia $\left(5^{\circ} 05^{\prime} \mathrm{N}\right.$ y $73^{\circ} 45^{\prime} \mathrm{W}$ ) a $2750 \mathrm{msnm}$. Los ejemplares que se utilizaron para las descripciones histológicas fueron once individuos con una longitud y peso total promedio de $22.01 \mathrm{~cm}$. y $92.70 \mathrm{~g}$. respectivamente. Para piel se tomaron muestras de seis zonas (cabeza dorsal, mandíbula, región dorsal, región media, región abdominal, región caudal) con un tamaño de $0.5 \mathrm{~cm}^{2}$ cada una. Las muestras se fijaron en formaldehído $4 \%$, se procesaron para inclusión en parafina, se obtuvieron cortes transversales de $5 \mu \mathrm{m}$ de espesor y se tiñeron con Hematoxilina-Eosina y PAS (Prophet 1995). Se realizaron observaciones con el microscopio de luz (Carl Zeiss Axioscop 2 Plus), posteriormente se realizó la captura de imágenes con la cámara CANON Power Shot G5. Como parámetros para la descripción histológica se definió: en la epidermis, el tipo de epitelio, componentes celulares especializados y estructuras sensoriales; en la dermis, el tipo de tejido conjuntivo, la organización de este tejido, y los componentes celulares típicos. Para la hipodermis se examinó el tipo de tejido, componentes celulares, además de la presencia de vascularización. Para la histomorfometría se emplearon las imágenes capturadas y el software de procesamiento y análisis de imágenes Scion Image PC (2000) de libre distribución en internet (www.scioncorp.com). Se midieron las siguientes variables en las seis zonas evaluadas: grosor de la epidermis y la dermis, número de células secretoras, número de estructuras sensoriales. Se establecieron algunos parámetros para la medición del grosor de capas: por cada corte evaluado se midieron en promedio tres puntos de la epidermis y tres de la dermis, que fueron promediados para obtener un dato único por zona de cada ejemplar; se midió la epidermis desde la parte superficial hasta el punto comprendido antes de la capa de melanóforos; y se realizaron mediciones de la dermis que incluyeron las dos capas de melanóforos que la flanquean. Los parámetros establecidos para el conteo de células secretoras y estructuras sensoriales fueron: células mucosas ovaladas o circulares, con un contorno bien definido; en las células clava se incluyeron dos tipos 
morfológicos, células circulares u ovaladas con vacuola conspicua y núcleo distinguible y células en forma de clava con vacuola conspicua y núcleo distinguible; para estructuras sensoriales (botones gustativos) debía ser evidente la forma de matraz característica; se midió el área transversal de la epidermis donde se contaron las células secretoras y botones gustativos para luego definir la cantidad de estas en un área de $50000 \mu \mathrm{m}^{2}$ con el fin de hacer comparables los datos obtenidos, que posteriormente se promediaron para obtener un dato único de cada variable por ejemplar. $\mathrm{La}$ metodología experimental planteada para piel corresponde a un diseño de bloques completos al azar con estructura de tratamientos factorial, donde se emplearon los datos obtenidos con la evaluación histomorfométrica y se analizaron con el software estadístico R-Project versión 2.1.1 de libre distribución en internet (www.r-project.org), con el cual se efectuaron pruebas de estadística: 1. Normalidad para definir si los datos evaluados presentaban una distribución normal, para lo cual se empleo el Test de Shapiro Wilk y los histogramas de distribución. 2. Análisis de Varianza ANOVA para reconocer si existían diferencias para las variables teniendo en cuenta factores como el tejido (dermis, epidermis), la zona (cabeza dorsal, mandíbula, región dorsal, región media, región abdominal, región caudal), sexo (hembra, macho). Para las células secretoras se utilizó un diseño similar mirando la cantidad de células de clava y mucosas dependiendo de la región y el sexo. A continuación se utilizó la prueba de Tukey Kramer para definir donde se establecían las diferencias detectadas con el ANOVA. Finalmente, se corroboraron las diferencias significativas obtenidas con el software calculando el intervalo de confianza al $95 \%$, que se determino luego de hallar el producto entre el error estándar de los datos y el valor de t (0.05 (2), n-1) (Zar 1999); para este caso se consideraron diferentes las variables en las cuales los intervalos no se traslapan. Este último procedimiento se realizó para dar mayor confiabilidad a los resultados.

\section{RESULTADOS}

A nivel macroscópico la piel de E. mutisii es lisa, sin escamas, de coloración variable, presentando manchas oscuras de forma serpenteante en la región dorsal y lateral, hacia la región ventral es de color claro con manchas más tenues. La organización tisular general no varió entre las zonas evaluadas. El tegumento de este pez consta de dos capas cutáneas principalmente (epidermis y dermis) y una subcutánea (hipodermis), como lo muestra la figura $1 \mathrm{~A}$.

La epidermis esta subdividida en tres estratos; el primero, conformado por células epiteliales definidas y algunas células mucosas; el segundo, conformado por células clava, algunas células mucosas y células epiteliales; el tercero, compuesto por células germinales indiferenciadas (Fig. 1B-C), adicionalmente la epidermis de E. mutisii presentó estructuras sensoriales como botones gustativos cuya morfología abarcó los tres estratos (Fig. 1D). Las células epiteliales se caracterizan por tener un tamaño pequeño en comparación con los otros tipos celulares. Sus núcleos presentan una coloración violeta intensa característica, la forma de estos varía según su posición en la epidermis (Fig. 1E): las células ubicadas cerca de la lámina basal presentan núcleos alargados u ovalados, característicos del estrato germinativo; las células que se encuentran en la parte superficial presentan núcleos aplanados que ocupan gran parte del citoplasma, debido a esta característica se otorga el nombre de epitelio plano estratificado; donde se observan aproximadamente de cuatro a cinco filas de estas células. Las células mucosas se caracterizan por tener un cuerpo esférico de gran tamaño, con citoplasma basófilo y un contorno delgado de color violeta, donde no fue posible distinguir el núcleo celular. Usualmente las células mucosas presentan una distribución regular en las capas superficial y media de la epidermis. La figura $1 \mathrm{~F}$ muestra el contenido glicoprotéico de estas células con la técnica de PAS, el cual se observa de color violeta intenso y de apariencia 

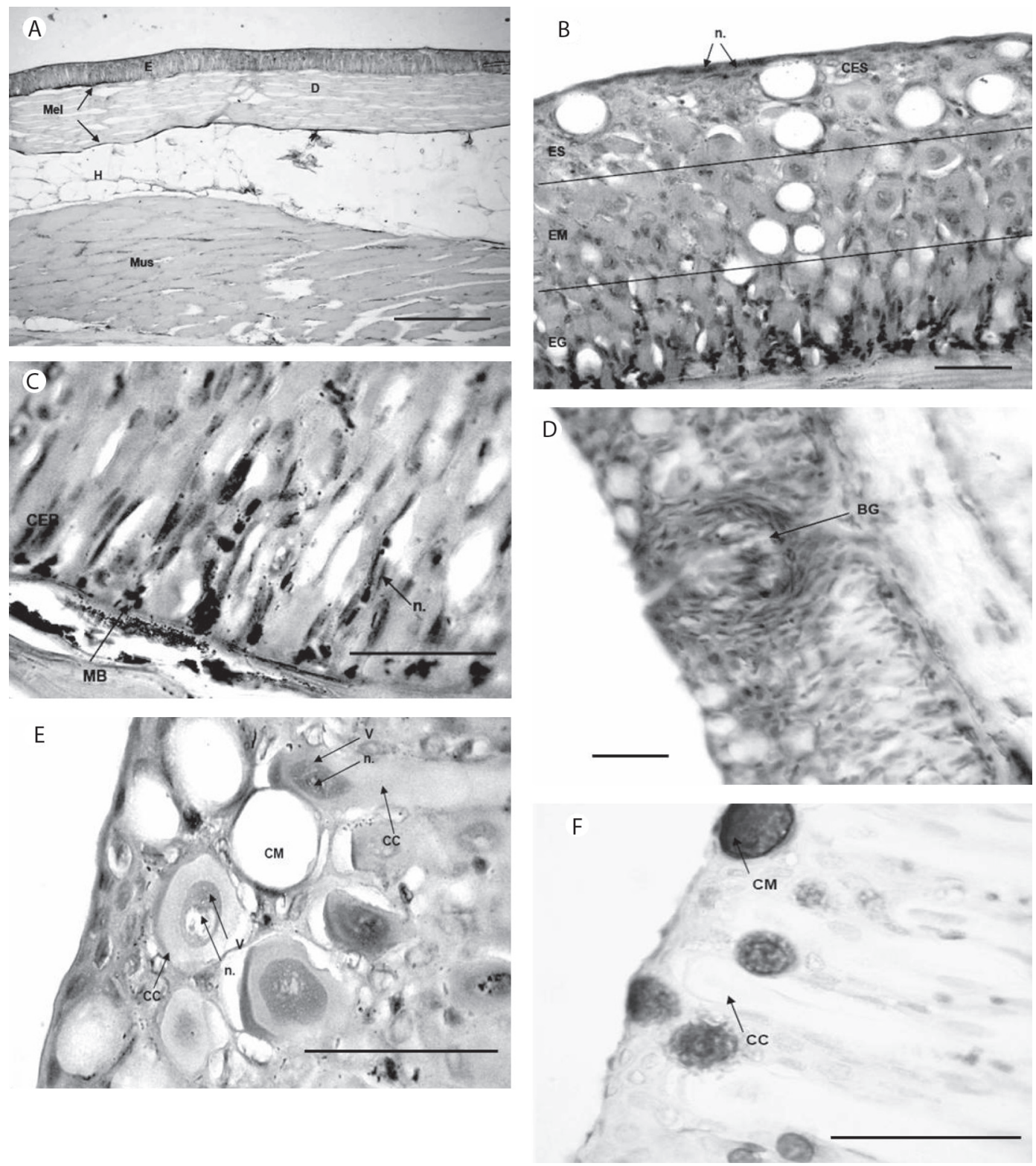

Fig. 1. Cortes transversales de piel de E. mutissi. A. Estructura general de la piel; epidermis (E); dermis (D); melanóforos (Mel); hipodermis $(\mathrm{H})$; músculo estriado (Mus) barra $=100 \mu$. B. Detalle de la epidermis. Estratos superficial (ES), medio (EM) y germinal (EG); células epiteliales superficiales (CES) y sus núcleos $(n))$ barra $=50 \mu$. C. Células epiteliales en la parte basal $(\mathrm{CEB})$, sus núcleos (n.) y la membrana basal $(\mathrm{MB})$ barra $=50 \mu$. D. Botón gustativo (BG) barra $=50 \mu$. E. Células clava (CC), vacuola (V), núcleo (n) 800X. F. Epidermis. Células mucosas (CM) y clava (CC). Tinción PAS. 800X.

Fig. 1. E. mutissi skin tranversal sections. A. General structure of the skin; epidermis (E); dermis (D); melanophores (Mel); hypodermis $(\mathrm{H})$; striated muscle (Mus). Bar $=100$ microns;. B. Epidermis details. Superficial layer (ES), medial layer(EM) and germinal layer (EG); superficial epithelial cells (CES) and its nuclei (n)) bar =

50 microns;. C. Basal epithelial cells (CEB) and its nuclei (n.) and basal membrane (MB) bar $=50$ microns;. D. Taste bud (BG) bar = 50 microns;. Club cells $(\mathrm{CC})$, vacuolae (V), nucleus (n) 800X. F. Epidermal mucous cells CM) and club cells CC). Staining PAS. $800 \mathrm{X}$. 
granular con un contorno celular bien definido. Las células clava se caracterizaron por presentar dos formas diferentes: a) cuerpo esférico y b) cuerpo en forma de clavo; para los dos casos se observó un citoplasma eosinófilo dentro del cual se presenta una vacuola conspicua de color rosado intenso que se ubica centralmente, (Fig. 1G).

Las células sensoriales quimiorreceptoras (que conforman los botones gustativos) se observaron en poca cantidad en la epidermis de la cabeza dorsal, mandíbula y regiones dorsal y abdominal (Fig.1D), con su porción apical en el estrato superficial y la porción basal en el estrato germinativo. Las células que conforman los neuromastos, característicos del epitelio de la cabeza y el tronco no se observaron en este estudio.

La dermis consiste de una capa de tejido conjuntivo denso regular; en la cual se pueden observar los núcleos de los fibroblastos de color violeta, posee dos filas de melanóforos (células de pigmento), las cuales flanquean la dermis; esta capa se observo pobremente vascularizada (Fig. 1H). La hipodermis se encuentra ubicada debajo de la dermis y esta compuesta principalmente de tejido adiposo (tejido conjuntivo especializado). Se observa que las células principales de este tejido son los adipocitos; estás células se encuentran en una malla violeta de tejido conjuntivo reticular, en el cual se pueden distinguir capilares sanguíneos.

Los datos morfométricos obtenidos muestran que la capa dérmica de E. mutisii presenta en promedio un mayor grosor (125.62 $\mu \mathrm{m}+/-10.38)$ que la capa epidérmica $(96.70$ $\mu \mathrm{m}+/-6.02, \mathrm{p}<0,01)$. E. mutisii presenta diferencias $(\mathrm{p}<0.01)$ en el grosor de la piel (epidermis + dermis) en la mandíbula (190.33 $\mu \mathrm{m}+/$-4.27) respecto al grosor de la piel en la región abdominal $(255.62 \mu \mathrm{m}+/-43.23)$, la cabeza dorsal $(212.64 \mu \mathrm{m}+/ 6.66)$ y la región dorsal $(240.18 \mu \mathrm{m}+/-36.88)$.

Se identificaron principalmente variaciones en el grosor de la capa dérmica entre zonas y variaciones en las dos capas cutáneas de una misma zona: el grosor de la dermis de la región abdominal (149.45 $\mu \mathrm{m}+/$-24.53) respecto al grosor de la dermis de la cabeza dorsal $(102.99 \mu \mathrm{m}+/-20.72)$ y la mandíbula $(93.03 \mu \mathrm{m}+/-23.78)$. El grosor de la dermis de la región media $(145.71 \mu \mathrm{m}+/$-26.98) respecto al grosor de la dermis de la mandíbula (93.03 $\mu \mathrm{m}+/$-23.78). Entre las dos capas cutáneas de la región abdominal (epidermis $106.17 \mu \mathrm{m}$ $+/ 18.49$ y dermis $149.45 \mu \mathrm{m}+/-24.53$ ) y de la región media (epidermis $83.41 \mu \mathrm{m}+/-11.79 \mathrm{y}$ dermis $145.71 \mu \mathrm{m}+/$-26.98).

El número de células secretoras en $E$. mutisii presenta una diferencia $(\mathrm{p}<0.01)$ que refleja una mayor cantidad de células clava (20 $+/$-2.68) respecto a la cantidad de células mucosas $(14+/-2.53)$ en un área de $50000 \mu \mathrm{m}^{2}$. La cantidad de células clava en la mandíbula (12 células/50 $000 \mu \mathrm{m}^{2}+/-4.55$ ) es diferente $(\mathrm{p}<0.01)$ respecto a la cantidad de estas mismas células en la región caudal (28 células/50 000 $\left.\mu \mathrm{m}^{2}+/ 11.25\right)$; la cantidad de células clava en la región media (26 células/50 $000 \mu \mathrm{m}^{2}+/-6.5$ ) respecto a la cantidad de células mucosas en la misma zona (8 células/50 $\left.000 \mu \mathrm{m}^{2}+/-6.58\right)$. Al calcular los intervalos de confianza, además de las diferencias anteriores se encontraron otras diferencias de esta interacción no detectadas en la prueba de Tukey: cantidad de células clava en la mandíbula (12 células/50 $\left.000 \mu \mathrm{m}^{2}+/-4.55\right)$ respecto a la cantidad de estas mismas células en la región dorsal (22 células $/ 50000 \mu \mathrm{m}^{2}$ $+/ 5.08$ ), región media (26 células/50 $000 \mu \mathrm{m}^{2}$ $+/-6.5$ ) y cabeza dorsal (21 células/50 $000 \mu \mathrm{m}^{2}$ $+/-4.33)$, cantidad de células clava en la región dorsal (22 células/50 $\left.000 \mu \mathrm{m}^{2}+/-5.08\right)$ respecto a la cantidad de células mucosas en la misma región (11 células/50 $\left.000 \mu \mathrm{m}^{2}+/-3.69\right)$.

El número de botones gustativos fue bajo. Para la región abdominal se contó el mayor número de estos $(0.19+/-0.19)$ y en las regiones caudal y media no se encontraron. Tampoco se observaron neuromastos.

\section{DISCUSIÓN}

La piel de E. mutisii esta compuesta por dos capas cutáneas y una subcutánea, comunes en la mayoría de peces teleósteos como lo 
muestra estudios realizados en diversas especies (Park.2000, Berraa y Humphrey 2002, Park 2002a, Park 2002b, Souza et al. 2003). Este estudio mostró que la piel se conforma por una epidermis delgada, compuesta de tejido epitelial, que se divide en tres estratos principalmente. Al interior de estos se observó una composición celular característica: en el estrato superficial predominan las células epiteliales y células mucosas; en el estrato medio se encuentran células clava principalmente y algunas células mucosas; finalmente, en el estrato germinativo se ubican células epiteliales indiferenciadas. Debido a que E. mutisii es un pez desprovisto de escamas, las primeras células en contacto con el medio, las células epiteliales superficiales, podrían contribuir en procesos de protección, cuando la piel sufre daños mecánicos o infecciones causadas por patógenos (Elliott 2000). En cuanto al estrato germinativo observado en la epidermis, no se distinguió una capa columnar o cúbica como lo sugiere la bibliografía (Elliott 2000), más bien este estrato se definió como indiferenciado, lo cual no afectaría la función de regeneración conocida para este estrato. La técnica de PAS permitió reconocer que el material de secreción de las células mucosas es de tipo glicoprotéico, posiblemente entre los componentes de estas secreciones se encuentren elementos antipatógenos y antimicrobianos que pueden prevenir la colonización de agentes extraños para evitar infecciones (Ottensen y Olafsen 1997).

La capacidad que presentan las células mucosas para almacenar gran cantidad de agua puede reflejar una adaptación importante de E. mutisii a su hábitat particular (sustrato fangoso, aguas poco profundas, entre otros) que ocasionalmente podría causarle desecación; de igual forma el mucus que estas secretan puede ayudar a la remoción de agentes extraños, patógenos y agentes intoxicantes (Takashi 1992). Finalmente, y no menos importante sería el papel que las secreciones de estas células puede cumplir en términos de protección en contra de la colonización de patógenos, la cual sería muy probable en E. mutisii debido al efecto de abrasión o fricción que tiene su cuerpo al ser este un pez bentónico con actividad generalmente nocturna (Cala et al. 1990).

De igual forma se pudo reconocer que el contenido vacuolar de las células clava es diferente al de las células mucosas, al no presentar la coloración violeta intensa característica de las glicoproteínas que reaccionan PAS. Algunos autores identifican las secreciones de estas células como una sustancia de alarma, o a asociarlas a las interacciones bióticas y reproducción (Chivers et al. 1996, Lebedeva et al. 1999) para lo cual serían necesarios seguimientos in vivo del capitán de la sabana con el fin de poder asociarlo con estos estudios.

Por otra parte, la información divulgada acerca de la función de estas células en la protección mecánica y/o antipatógena es limitada. Entre los pocos estudios esta el realizado por Michalak (2005) donde concluye que las células clava incrementan su número con el fin de evitar la entrada de patógenos y parásitos a la piel, lo anterior podría asociarse al caso particular de E. mutisii ya que se observó predominancia de este tipo de células secretoras en las zonas de piel evaluadas y como se mencionó anteriormente este pez pude estar propenso a afecciones cutáneas causadas por el medio en que habita. La abundancia de estas células también puede resultar en una ayuda mecánica para resistir la presión del agua, lo cual permite sugerir que la cantidad de células se relaciona con el soporte hidrostático.

Uno de los objetivos de este estudio era reconocer estructuras que permitieran asociar la piel a funciones sensoriales. Se esperaba encontrar este tipo de estructuras sensoriales en las muestras de piel evaluadas. Sin embargo los resultados histológicos indicaron la presencia de pocos botones gustativos y una posible ausencia de neuromastos superficiales, por lo cual no es posible atribuir funciones sensoriales determinantes en la piel de E. mutisii. Los botones gustativos se presentaron con una baja densidad en la piel, a pesar de estos resultados no se puede desconocer su función sensorial sugiriendo que pueden contribuir a la búsqueda de alimento, ya que se ubicaron principalmente hacia la superficie dorsal y ventral. Además, 
se podría considerar como estrategia para $E$. mutisii ocupar menor área para desempeñar un papel gustativo, presentado pocos botones que sean lo suficientemente desarrollados para la recepción de señales, lo cual solo podría confirmarse a través de análisis ultraestructurales y de fuentes de innervación. Por otra parte, la presencia de neuromastos se asocia de forma directa con el sistema de línea lateral en los peces (sistema mecanoreceptor o hidrodinámico) que contribuye en los comportamientos que incluyen alimentación, comunicación, exploración del medio y orientación asociada con las corrientes (Coombs 2001). De igual forma, otros estudios (Kotrschal et al. 1998, Pohlmann 2004) han demostrado que este sistema es muy desarrollado en peces que habitan sitios oscuros, que carecen de visión y principalmente con una dieta piscívora. Las condiciones de poca visibilidad (por hábitat y ojos involucionados) a las cuales se ha adaptado el capitán de la sabana, permitirían inferir que requiere de un sistema de línea lateral desarrollado que le permita desenvolverse en el medio, sin embargo en observaciones morfológicas a nivel macroscópico de los individuos empleados para este estudio no se evidencio la línea lateral, lo cual podría estar relacionado con la ausencia de neuromastos libres o poros que permitan la comunicación del canal con el exterior en las muestras de piel evaluadas. Lo anterior no refleja una carencia de este sistema en E. mutisii, puesto que en uno de los ejemplares empleados por Contreras y Tovar (en preparación) se observó el canal interno de la línea lateral, que se asocia a la función mecanoreceptora.

En cuanto a la dermis de E. mutisii, las observaciones histológicas no muestran una clara división de los estratos esponjoso y compacto, por lo cual se definió únicamente como tejido conjuntivo denso regular. Esto sugiere que la dermis soportaría la presión ejercida por el medio físico (asociado con el sustrato) actuando como capa amortiguadora. En cuanto a la vascularización de la dermis descrita en otros teleósteos (Berraa y Humphrey 2002, Park 2002a, Park 2002b, Souza et al. 2003, Le
Guellec et al. 2004); se puede decir que E. mutisii difiere levemente, al presentar poca vascularización que se puede atribuir a un bajo nivel metabólico o posiblemente a que la dermis presenta mucho colágeno. Otra particularidad de las observaciones histológicas fue encontrar células de pigmento que corresponden al grupo de melanóforos flanqueando la dermis. Aunque no se realizó un conteo de estas células fue evidente la abundancia hacia las superficies dorsal y lateral del pez, lo cual se puede asociar con la pigmentación característica observada microscópicamente; estas condiciones crípticas se pueden atribuir al medio en que habita y a una adaptación propia a la evasión de depredadores (Parichy y Turner 2002).

Los resultados a nivel histológico para la piel de E. mutisii se inclinan a asignarle una función protectora y de soporte para este pez debido a la conformación de las capas cutáneas y a las células encontradas en la epidermis.

Los resultados morfométricos reflejaron diferencias significativas entre el grosor de las dos capas cutáneas donde la dermis es la capa mas gruesa, lo cual sumado a la rigidez otorgada por el tipo de tejido, como se menciono anteriormente, puede hacer que esta capa cumpla funciones como mantener la firmeza y resistir la torsión del cuerpo cuando el pez esta en movimiento (Long et al. 1996). A pesar que únicamente hay diferencias significativas en las regiones abdominal y media, este resultado permite confirmar las funciones mencionadas en el párrafo anterior puesto que: es probable que la región media requiera de una dermis mas gruesa que le permita tener mayor flexibilidad y soportar así la fuerza de los movimientos propios de la actividad de nado. Por otra parte, en la región abdominal y por ende la mas expuesta al contacto con el medio físico la capa dérmica puede contribuir a tolerar la presión mecánica directa. Por otra parte, el grosor de la piel (epidermis + dermis) de E. mutisii en la mandíbula es significativamente menor al registrado en las regiones abdominal, dorsal y cabeza dorsal, lo cual puede ser explicado por la estructura de la mandíbula que a nivel macroscópico reflejo ser muy delgada y compacta; sin embargo, el grosor 
en la piel de la mandíbula no se puede asumir como una zona ventral desprovista de protección puesto que cuenta con numerosas células mucosas. Finalmente los resultados morfométricos, de las capas, mostraron que el grosor de la epidermis no varia significativamente según la zona piel evaluada, reflejando una uniformidad de esta capa en el cuerpo del pez.

En el caso particular de la densidad y distribución de las células secretoras, los resultados morfométricos permitieron detectar diferencias significativas en la cantidad de células mucosas en relación con las células clava presentes en un área de $50000 \mu \mathrm{m}^{2}$ de piel (corte transversal) de E. mutisii. Por la mayor cantidad de células clava, se podría inferir que estas células desempeñan un papel importante en el sistema inmune del organismo (Michalak 2005) como eventual respuesta a lesiones mecánicas y/o por patógenos. Los resultados de la evaluación del número de células secretoras según la zona de piel, muestran que hay variaciones significativas en la densidad de las células clava entre la región caudal y la mandíbula, lo cual se podría explicar porque la mandíbula al presentar una piel delgada puede establecer como mecanismo de protección la generación de secreciones por parte de las células mucosas, implicando así una mayor cantidad, para mantenerse lubricada. Otra diferencia encontrada se ubico particularmente en la región media, donde la cantidad de células clava es mayor que la de células mucosas, esto se puede relacionar con la función de resistencia que puede complementarse con el grosor de la dermis en esta zona. Finalmente, no hay diferencias en la densidad de células mucosas a lo largo del cuerpo, por lo tanto esta distribución regular podría garantizar una capa de mucus homogénea sobre la superficie del pez (Elliott 2000), que asegura la protección de la piel.

\section{AGRADECIMIENTOS}

Agradecemos a la Universidad Militar Nueva Granada por la financiación de este trabajo de investigación.

\section{RESUMEN}

Se estudió la piel del pez dulceacuícola endémico de Colombia Eremophylus mutissi. Se tomaron muestras de piel $\left(0.5 \times 0.5 \mathrm{~cm}^{2}\right)$ de 11 especimenes en seis partes del cuerpo (mandíbulas, cabeza dorsal, tronco dorsal, tronco caudal, tronco medial y abdominal). Se fijaron en formaldehído al $4 \%$, con deshidratación en etanol al $95 \%$ e isopropanol al $99 \%$, inclusión en parafina y cortes a $5 \mu \mathrm{m}$. La piel está constituida por dos capas cutáneas (epidermis y dermis) y una capa subcutánea (hipodermis): la epidermis tiene tres capas con células secretoras, células epiteliales y pocas células gustativas; la dermis está separada de la epidermis por una membrana basal. Observamos fibroblastos, dos capas de melanóforos y algunos vasos sanguíneos; la hipodermis tiene un tejido adiposo vascularizado. La dermis es más delgada que la epidermis; la piel tiene más células tipo clava que células mucosas. El tronco medio tiene muchas células clava y células mucosas. La piel de E. mutissi parece tener una función principalmente protectora.

Palabras clave: E. Mutissi, piel, histología, morfometría.

\section{REFERENCIAS}

Berraa, T.M. \& J.D. Humphrey. 2002. Gross anatomy and histology of the hook and skin of forehead brooding male nurseryfish, Kurtus gulliveri, from northern Australia. Env. Biol. Fishes. 65:263-270.

Bonilla, R.J., M. Quintero \& H. Hurtado. 2005. Estudio histológico preliminar de piel y barbillones del pez capitán de la sabana Eremophilus mutisii (Humboldt, 1805). Revista Facultad de Ciencias de la Universidad Militar Nueva Granada. 1:75-77.

Borissow, C. \& A. Canosa. 2000. Aislamiento e identificación de las bacterias presentes en lesiones externas e internas del capitán de la sabana, Eremophilus mutisii (Humboldt, 1805). Geotrópica 5:5-14.

Cala, P., B. del Castillo \& B. Garzón. 1990. Air-breathing behaviour of the Colombian catfish Eremophilus mutisii (Trichomycteridae, Siluriformes). Exp. Biol. 48:357-360.

Coombs, S., C.B. Braun \& B. Donovan. 2001. The orienting response of lake Michigan mottled sculpin is mediated by canal neuromasts. J. Exp. Biol. 204:337-348.

Chivers, D.P., G.E. Brown \& R.J.F. Smith. 1996. The evolution of chemical alarm signals: Attracting predators benefits alarm signal senders. Am. Nat. 148:649-659. 
Elliottt, D.G. 2000. Integumentary System. p. 271-300. In G. Ostrander. The Laboratory Fish. Academic, Washington, DC, USA.

Grazzle, J. 1976. Anatomy and Histology of the Channel Catfish. Agricultural Experiment Station. Auburn University, Alabama, USA. 94 p.

Jiménez, J.A. \& G.J. Pinto. 2005. Aspectos biológicos del capitán de la sabana Eremophilus mutisii (Humboldt, 1805) (pisces) de un tramo del río Bogotá en el municipio de Suesca (Cundinamarca), Colombia. Trabajo grado, Universidad Militar Nueva Granada, Bogotá D.C. 173 p.

Kotrschal, K., M.J. Van Staaden \& R. Huber. 1998. Fish brains: evolution and environmental relationships. Rev. Fish Biol. Fisher. 8: 373-408.

Lebedeva, N.E., M.Z. Vosyliene, L.A. Petrauskiene \& T.V. Golovkina. 1999. Alarm substances in rainbow trout: Origin and physiological effects. Pheromones 6: 61-64.

Le Guellec, D., G. Morvan-Dubois \& J.Y. Sire. 2004. Skin development in bony fish with particular emphasis on collagen deposition in the dermis of the zebrafish (Danio rerio). J. Dev. Biol. 48:217-231.

Long, J.H., M.E. Hale, M.J. McHenrry \& M.W. Westneat. 1996. Functions of fish skin: flexural stiffnes and steady swimming of longnose gas Lepisosteus osseus. J. Exp. Biol. 199:2139-2151

Mayorga, M. 1992. Biología reproductiva y alimentación de las poblaciones de capitán de la Sabana Eremophilus mutisii, Humbolt 1805 (pisces Trichomycteridae), en la laguna de Fúquene. Tesis Facultad de Biología Marina, Universidad Jorge Tadeo Lozano, Bogotá, Colombia. $80 \mathrm{p}$.

Michalak, T. 2005. The effects of pathogens, parasites, and familiarity on alarm cell investment in fathead minnows, Pimephales promelas. Thesis for the degree of Masters of Science in the department of biology University of Saskatchewan, Saskatoon, Canada. 77p.

Ottensen, O.H. \& J.A. Olafsen. 1997. Ontogenetic development and composition of the mucous cells and the occurrence of succular cells in the epidermis of Atlantic halibut. J. Fish Biol. 50:620-633.

Parichy, D.M. \& J.M. Turner. 2002. Temporal and cellular requirements for Fms signaling during zebrafish adult pigment pattern development. Develop. 130: 817-833.
Park, J.Y. 2002a. Morphology and histochemistry of the skin of the Korean spined loach, Iksookimia koreensis (Cobitidae), in relation to respiration. Folia Zool. 51: 241-247.

Park, J.Y. 2002b. Structure of the skin of an air-breathing mudskipper, Periophthalmus magnuspinnatus. J. Fish Biol. 60: 1543-1550.

Park, J.Y., I.S. Kim \& S.Y. Kim. 2000. Histology of skin of the amphibious fish Periophtalmus modestus. Korean J. Biol. Sci. 4: 315-318.

Pohlmann, K., J. Atema \& T. Breithaupt. 2004. The importance of the lateral line in nocturnal predation of piscivorous catfish. J. Exp. Biol. 207: 2971-2978.

Prophet, E. 1995. Procesamiento de tejidos: deshidratación, aclaración e infiltración, p 31-332 In E.B. Prophet, B. Mills, J.B. Arrington, L.H. Sobin (eds). Métodos histotecnológicos. Washington, Washington. USA.

Rodríguez, A. 1991. Evaluación de los cambios patológicos originados durante el cultivo experimental del capitán de la sabana (Eremophilus mutisii, Humboldt 1805) en la estación piscícola del Neusa (CAR). Bogotá, Cundinamarca, Colombia.

Rodríguez, A. 2000. El pez "Capitán del a Sabana", Eremophilus mutisii, en el Altiplano Cundiboyacense. Colombia Ciencia y Tecnol. 18: 38-40.

Souza, M.L.R., DM. Dourado, S.D. Machado, D.F. Buccini, M.I. A. Jardim, R. Matias, C. Correia \& I.C. Ferreira. 2003. Análise da pele de três espécies de peixes: histologia, morfometria e testes de resistência. Rev. Bras. Zootec. 32: 1551-1559.

Stone, L.M., T.E. Finger, P.P. Tam \& S.S. Tan. 1995. Taste receptor cells arise from local epithelium, not neurogenic ectoderm. Proc. Nat. Acad. Sci. 92: 1916-1920.

Takashi, H. 1992. An atlas of fish histology. Normal and pathology features. Kodansha Tokyo, Japón.

Tyagi, R. \& A.N. Shukla. 2002. Encyclopedia of fishes. Series anatomy of fishes. Fifth Edition. Nueva Delhi, India, 181-187p.

Webb, J.F. 2000. Mechanosensory lateral line: Microscopic anatomy and development, p. 463-468. In G. Ostrander. The Laboratory Fish. Academic, Washington, USA.

Zar, J.H. 1999. Biostatistical Analysis. Prentice Hall. Londres, Inglaterra, p. 117-272. 